\title{
GB-splines of arbitrary order ${ }^{1}$
}

\author{
Boris I. Ksasov ${ }^{\mathrm{a}, *}$, Pairote Sattayatham ${ }^{\mathrm{b}}$ \\ ${ }^{a}$ Institute of Computational Technologies, Russian Academy of Sciences, Lavrentyev Avenue 6, 630090, \\ Novosibirsk, Russia \\ ${ }^{b}$ School of Mathematics, Suranaree University of Technology, University Avenue 111, 30000, \\ Nakhon Ratchasima, Thailand
}

Received 6 April 1998; received in revised form 23 October 1998

\begin{abstract}
Explicit formulae and recurrence relations for the calculation of generalized B-splines (GB-splines) of arbitrary order are given. We derive main properties of GB-splines and their series, i.e. partition of unity, shape-preserving properties, invariance with respect to aftine transformations, etc. It is shown that such splines have the variation diminishing property and are Chebyshevian splines. (C) 1999 Elsevier Science B.V. All rights reserved.
\end{abstract}

Keywords: Spline; GB-spline; Weak Chebyshevian system; Variation diminishing; Shape-preserving approximation

\section{Introduction}

Fitting curves and surfaces to functions and data requires the availability of methods which preserve the shape of the data. In practical calculations, we usually deal with data given with prescribed accuracy. Therefore, we need to develop methods for constructing fair-shape-preserving approximations that satisfy given tolerances and inherit major geometric properties of the data such as positivity, monotonicity, convexity, presence of linear sections, etc. Such approximations, based on GB-splines [12] with automatic choice of tension parameters are suggested in [11].

Until recently, local support bases for computations with generalized splines have been available only for some special types of splines [3, 13, 15]. This limits the choice of methods when using generalized splines. In [7-9] local support basis functions for exponential splines were introduced and their application to interpolation problems was considered. A recurrence relation for rational B-splines with prescribed poles was obtained in [5]. In [10, 12] one of the authors constructed

\footnotetext{
* Corresponding author. E-mail: boris@math.sut.ac.th.

${ }^{1}$ Supported by grant BRG/16/2540 of the Thailand Research Fund.
} 
GB-splines for tension generalized splines allowing the tension parameters to vary from interval to interval.

In this paper, we expand the main results in [12] to GB-splines of arbitrary order. These GB-splines are nonnegative functions with supports of minimal length which form a partition of unity. We get explicit formulae for such GB-splines and develop recurrence algorithms for their calculation. In the particular case of polynomial B-splines, we recover the well-known recurrence relation for such B-splines [1]. The main properties of GB-splines and their series such as shapepreserving properties, invariance with respect to affine transformations, etc., are investigated. It is shown that the GB-spline series is a variation diminishing function and the systems of GB-splines are weak Chebyshevian systems.

\section{GB-splines of arbitrary degree}

Let a partition $\Delta: a=x_{0}<x_{1}<\cdots<x_{N}=b$ of the interval $[a, b]$ be given to which we associate a space of splines $S_{n}^{G}$ whose restriction to a subinterval $\left[x_{i}, x_{i+1}\right], i=0, \ldots, N-1$ is spanned by the system on linearly independent functions $\left\{1, x, \ldots, x^{n-3}, \Phi_{i, n}, \Psi_{i, n}\right\}, n \geqslant 2$, and where every function in $S_{n}^{G}$ has $n-2$ continuous derivatives.

Definition 2.1. A generalized spline of order $n$ is a function $S \in S_{n}^{G}$ such that

(i) for any $x \in\left[x_{i}, x_{i+1}\right], i=0, \ldots, N-1$,

$$
S(x)=P_{i, n-2}(x)+S^{(n-2)}\left(x_{i}\right) \Phi_{i, n}(x)+S^{(n-2)}\left(x_{i+1}\right) \Psi_{i, n}(x),
$$

where $P_{i, n-2}$ is a polynomial of order $n-2$, and

$$
\begin{aligned}
& \Phi_{i, n}^{(r)}\left(x_{i+1}\right)=\Psi_{i, n}^{(r)}\left(x_{i}\right)=0, \quad r=0, \ldots, n-2, \\
& \Phi_{i, n}^{(n-2)}\left(x_{i}\right)=\Psi_{i, n}^{(n-2)}\left(x_{i+1}\right)=1
\end{aligned}
$$

(ii) $S \in C^{n-2}[a, b]$.

The functions $\Phi_{i, n}$ and $\Psi_{i, n}$ depend on tension parameters. In practice, we choose $\Phi_{i, n}(x)=\Phi_{i, n}\left(p_{i}, x\right)$, $\Psi_{i, n}(x)=\Psi_{i, n}\left(q_{i}, x\right), 0 \leqslant p_{i}, q_{i}<\infty$. In the limiting case when $p_{i}, q_{i} \rightarrow \infty$ we require that $\lim _{p_{i} \rightarrow \infty}$ $\Phi_{i, n}\left(p_{i}, x\right)=0, x \in\left[x_{i}, x_{i+1}\right]$ and $\lim _{q_{i} \rightarrow \infty} \Psi_{i, n}\left(q_{i}, x\right)=0, x \in\left[x_{i}, x_{i+1}\right]$ so that the function $S$ in formula (1) turns into a polynomial of the order $n-2$. Additionally, we require that if $p_{i}=q_{i}=0$ for all $i$ we get a conventional polynomial spline of degree $n$ with $\Phi_{i, n}(x)=-\frac{\left(x-x_{i+1}\right)^{n-1}}{(n-1) ! h_{i}}, \Psi_{i, n}(x)=\frac{\left(x-x_{i}\right)^{n-1}}{(n-1) ! h_{i}}, h_{i}=x_{i+1}-x_{i}$.

Consider now the problem of constructing a basis in the space $S_{n}^{G}$ consisting of functions with local supports of minimal length. For this, it is convenient to extend the mesh $\Delta$ by adding points $x_{-n+1}<\cdots<x_{-1}<a, b<x_{N+1}<\cdots<x_{N+n-1}$. As $\operatorname{dim}\left(S_{n}^{G}\right)=(n) N-(n-1)(N-1)=N+n-1$, it is sufficient to construct a system of linearly independent GB-splines $B_{j, n}, j=-n+1, \ldots, N-1$, in $S_{n}^{G}$ such that $B_{j, n}(x)>0$ if $x \in\left(x_{j}, x_{j+n}\right)$ and $B_{j, n} \equiv 0$ outside $\left(x_{j}, x_{j+n}\right)$.

For $n>2$ we require the fulfillment of the normalization condition

$$
\sum_{j=-n+1}^{N-1} B_{j, n}(x) \equiv 1 \quad \text { for } x \in[a, b] \text {. }
$$


According to (1), on the interval $\left[x_{j+l}, x_{j+l+1}\right], l=0, \ldots, n-1$, the GB-spline $B_{j, n}$ has the form

$$
B_{j, n}(x)=P_{j, l, n-2}(x)+B_{j, n}^{(n-2)}\left(x_{j+l}\right) \Phi_{j+l, n}(x)+B_{j, n}^{(n-2)}\left(x_{j+l+1}\right) \Psi_{j+l, n}(x)
$$

where $P_{j, l, n-2}$ is a polynomial of order $n-2$.

Taking into account the continuity conditions for neighboring polynomials $P_{j, l-1, n-2}$ and $P_{j, l, n-2}$, $l=1, \ldots, n-1$, in (4), we have the relations

$$
P_{j, l, n-2}(x)=P_{j, l-1, n-2}(x)+B_{j, n}^{(n-2)}\left(x_{j+l}\right) \sum_{r=0}^{n-3} z_{j+l, n}^{(r)}\left(x-x_{j+l}\right)^{r} / r !, \quad l=1, \ldots, n-1
$$

with $z_{j+l, n}^{(r)}=\Psi_{j+l-1, n}^{(r)}\left(x_{j+l}\right)-\Phi_{j+l, n}^{(r)}\left(x_{j+l}\right), r=0, \ldots, n-3$.

As $B_{j, n}(x)=0$ if $x \notin\left(x_{j}, x_{j+n+2}\right)$ and by (5), the polynomials $P_{j, l, n-2}$ are identical to zero when $l=0$ and $l=n$. Then by repeated application of formula (5) we have

$$
\begin{aligned}
P_{j, l, n-2}(x) & =\sum_{l^{\prime}=1}^{l} B_{j, n}^{(n-2)}\left(x_{j+l^{\prime}}\right) \sum_{r=0}^{n-3} z_{j+l^{\prime}, n}^{(r)}\left(x-x_{j+l^{\prime}}\right)^{r} / r ! \\
& =-\sum_{l^{\prime}=l+1}^{n} B_{j, n}^{(n-2)}\left(x_{j+l^{\prime}}\right) \sum_{r=0}^{n-3} z_{j+l^{\prime}, n}^{(r)}\left(x-x_{j+l^{\prime}}\right)^{r} / r !, \quad l=1, \ldots, n-2 .
\end{aligned}
$$

In particular, the following identity is valid:

$$
\sum_{l=1}^{n-1} B_{j, n}^{(n-2)}\left(x_{j+l}\right) \sum_{r=0}^{n-3} z_{j+l, n}^{(r)}\left(x-x_{j+l}\right)^{r} / r ! \equiv 0 .
$$

Using the expansion of polynomials by powers of $x$ we can rewrite (7) in the form

$$
\sum_{l=1}^{n-1} B_{j, n}^{(n-2)}\left(x_{j+l}\right) \sum_{\alpha=0}^{n-3} \frac{x^{\alpha}}{\alpha !} \sum_{r=\alpha}^{n-3} z_{j+l, n}^{(r)} \frac{\left(-x_{j+l}\right)^{r} \alpha}{(r-\alpha) !} \equiv 0 .
$$

Now by equating the coefficients of the monomials $x^{\alpha}, \alpha=0,1, \ldots, n-3$, in (8) to zero, we arrive at a system of $n-2$ linear algebraic equations which defines the unknown quantities $B_{j, n}^{(n-2)}\left(x_{j+l}\right)$, $l=1, \ldots, n-1$,

$$
\sum_{l=1}^{n} B_{j, n}^{(n-2)}\left(x_{j+l}\right) \sum_{r=\alpha}^{n} z_{j+l, n}^{(r)} \frac{\left(-x_{j+l}\right)^{r-\alpha}}{(r-\alpha) !}=0, \quad \alpha=0, \ldots, n-3 .
$$

To obtain the unique solution of this system we can use the normalization condition (3). Substituting formula (4) into the identity (3) written for $x \in\left[x_{i}, x_{i+1}\right]$, we obtain

$$
\sum_{j=i-n+1}^{i} B_{j, n}(x)=\Phi_{i, n}(x) \sum_{j=i-n+1}^{i-1+1} B_{j, n}^{(n-2)}\left(x_{i}\right)+\Psi_{i, n}(x) \sum_{j=i-n+2}^{i} B_{j, n}^{(n-2)}\left(x_{i+1}\right)+\sum_{j=i-n+2}^{i-1} P_{j, i-j, n-2}(x) \equiv 1 .
$$

Since according to (3)

$$
\sum_{j=i-n+1}^{i-1} B_{j, n}^{(n-2)}\left(x_{i}\right)=\sum_{j=i-n+2}^{i} B_{j, n}^{(n-2)}\left(x_{i+1}\right)=0,
$$


it follows from (6) that

$$
\sum_{j=i-n+2}^{i-1} P_{j, i-j, n-2}(x)=\sum_{j=i-n+2}^{i-1} \sum_{l=1}^{i-j} B_{j, n}^{(n-2)}\left(x_{j+l}\right) \sum_{r=0}^{n-3} z_{j+l, n}^{(r)}\left(x-x_{j+l}\right)^{r} / r ! \equiv 1 .
$$

This gives us the system of linear equations

$$
\sum_{j=i-n+2}^{i-1} \sum_{l=1}^{i-j} B_{j, n}^{(n-2)}\left(x_{j+l}\right) \sum_{r=\alpha}^{n-3} z_{j+l, n}^{(r)} \frac{\left(-x_{j+l}\right)^{r-\alpha}}{(r-\alpha) !}=\delta_{0, \alpha}, \quad \alpha=0, \ldots, n-3,
$$

where $\delta_{0, \alpha}$ is the Kronecker symbol.

We can eliminate the unknowns analogously as has been done in $[10,12]$. Having computed the unknowns $B_{j, n}^{(n-2)}\left(x_{j+l}\right), l=1, \ldots, n-1$, we find the coefficients of the polynomials $P_{j, l, n-2}, l=$ $1, \ldots, n-2$, in (4) by using formulae (6). In this way, the computation of the coefficients of the polynomials $P_{j, l, n-2}$ can be realized starting from either the left or right endpoint of the support interval.

\section{Recurrence algorithm for the calculation of GB-splines}

Let us define the function

$$
B_{j, 2}(x)= \begin{cases}\Psi_{j, n}^{(n-2)}(x), & x_{j} \leqslant x \leqslant x_{j+1}, \\ \Phi_{j+1, n}^{(n-2)}(x), & x_{j+1} \leqslant x \leqslant x_{j+2}, \\ 0, & x \notin\left(x_{j}, x_{j+2}\right),\end{cases}
$$

where the functions $\Psi_{j, n}^{(n-2)}$ and $\Phi_{j+1, n}^{(n-2)}$ are assumed to be positive and monotone on $\left(x_{j}, x_{j+1}\right)$ and $\left(x_{j+1}, x_{j+2}\right)$ respectively.

We will consider the sequence of GB-splines defined by the recurrence formula

$$
B_{j, k}(x)=\int_{x_{j}}^{x} \frac{B_{j, k-1}(\tau)}{c_{j, k-1}} \mathrm{~d} \tau-\int_{x_{j+1}}^{x} \frac{B_{j+1, k-1}(\tau)}{c_{j+1, k-1}} \mathrm{~d} \tau, \quad k=3, \ldots, n,
$$

where

$$
c_{j, k-1}=\int_{x_{j}}^{x_{j+k-1}} B_{j, k-1}(\tau) \mathrm{d} \tau
$$

In practical calculations, an alternate representation of formula (10),

$$
B_{j, k}(x)=-\int_{x}^{x_{j+k-1}} \frac{B_{j, k-1}(\tau)}{c_{j, k-1}} \mathrm{~d} \tau+\int_{x}^{x_{j+k}} \frac{B_{j+1, k}(\tau)}{c_{j+1, k-1}} \mathrm{~d} \tau, \quad k=3, \ldots, n
$$

is useful.

By differentiating formula (10) we obtain

$$
B_{j, k}^{\prime}(x)=B_{j, k-1}(x) / c_{j, k-1}-B_{j+1, k-1}(x) / c_{j+1, k-1}, \quad k=3, \ldots, n .
$$


Theorem 3.1. The recurrence formulae (9) and (10) define the sequence of GB-splines of the form

$$
B_{j, k}(x)= \begin{cases}B_{j, k}^{(k-2)}\left(x_{j+1}\right) \Psi_{j, n}^{(n-k)}(x), & x_{j} \leqslant x \leqslant x_{j+1}, \\ P_{j, l, k-2}(x)+B_{j, k}^{(k-2)}\left(x_{j+l}\right) \Phi_{j+l, n}^{(n-k)}(x) & \\ \quad+B_{j, k}^{(k-2)}\left(x_{j+l+1}\right) \Psi_{j+l, n}^{(n-k)}(x) & x_{j+l} \leqslant x \leqslant x_{j+l+1}, l=1, \ldots, k-2, \\ B_{j, k}^{(k-2)}\left(x_{j+k-1}\right) \Phi_{j+k, n}^{(n-k)}(x), & x_{j+k-1} \leqslant x \leqslant x_{j+k}, \\ 0, & x \notin\left(x_{j}, x_{j+k}\right),\end{cases}
$$

$k=2, \ldots, n$, where

$$
\begin{aligned}
P_{j, l, k-2}(x) & =\sum_{l^{\prime}=1}^{l} B_{j, k}^{(k-2)}\left(x_{j+l^{\prime}}\right) \sum_{r=n-k}^{n-3} z_{j+l^{\prime}, n}^{(r)}\left(x-x_{j+l^{\prime}}\right)^{r-n+k} /(r-n+k) ! \\
& =-\sum_{l^{\prime}=l+1}^{k-1} B_{j, k}^{(k-2)}\left(x_{j+l^{\prime}}\right) \sum_{r=n-k}^{n-3} z_{j+l^{\prime}, n}^{(r)}\left(x-x_{j+l^{\prime}}\right)^{r-n+k} /(r-n+k) !
\end{aligned}
$$

and

$$
\sum_{l=1}^{k-1} B_{j, k}^{(k-2)}\left(x_{j+l}\right) \sum_{r=n-k}^{n-3} z_{j+l, n}^{(r)}\left(x-x_{j+l}\right)^{r-n+k} /(r-n+k) ! \equiv 0, \quad k=3, \ldots, n .
$$

Proof. For $k=2$ the formula (12) takes the form

$$
B_{j, 2}(x)= \begin{cases}B_{j, 2}\left(x_{j+1}\right) \Psi_{j, n}^{(n-2)}(x), & x_{j} \leqslant x \leqslant x_{j+1}, \\ B_{j, 2}\left(x_{j+1}\right) \Phi_{j+1, n}^{(n-2)}(x), & x_{j+1} \leqslant x \leqslant x_{j+2}, \\ 0, & x \notin\left(x_{j}, x_{j+2}\right) .\end{cases}
$$

We choose $B_{j, 2}\left(x_{j+1}\right)=1$, and then by (2), this formula coincides with (9).

Using mathematical induction, we assume that the assertion of the theorem is fulfilled for some $k^{\prime}=k-1<n-1(k=3, \ldots, n-1)$. Let us show its validity for $k \leqslant n$. According to (10) and (12) we have

$$
\begin{aligned}
& B_{j, k}(x)=\frac{1}{c_{j, k^{\prime}}} B_{j, k^{\prime}}^{\left(k^{\prime}-2\right)}\left(x_{j+1}\right) \Psi_{j, n}^{\left(n-k^{\prime}-1\right)}(x), \quad x \in\left[x_{j}, x_{j+1}\right], \\
& B_{j, k}(x)=-\frac{1}{c_{j+1, k^{\prime}}} B_{j+1, k^{\prime}}^{\left(k^{\prime}-2\right)}\left(x_{j+k^{\prime}}\right) \Phi_{j+k^{\prime}, n}^{\left(n-k^{\prime}-1\right)}(x), \quad x \in\left[x_{j+k^{\prime}}, x_{j+k^{\prime}+1}\right] .
\end{aligned}
$$

By virtue of (11) and because GB-splines have local supports,

$$
\begin{aligned}
& B_{j, k}^{(k-2)}\left(x_{j+1}\right)=B_{j, k^{\prime}}^{\left(k^{\prime}-2\right)}\left(x_{j+1}\right) / c_{j, k^{\prime}}, \\
& B_{j, k}^{(k-2)}\left(x_{j+k-1}\right)=-B_{j+1, k^{\prime}}^{\left(k^{\prime}-2\right)}\left(x_{j+k^{\prime}}\right) / c_{j+1, k^{\prime}} .
\end{aligned}
$$


Therefore,

$$
\begin{aligned}
& B_{j, k}(x)=B_{j, k}^{(k-2)}\left(x_{j+1}\right) \Psi_{j, n}^{(n-k)}(x), \quad x \in\left[x_{j}, x_{j+1}\right], \\
& B_{j, k}(x)=B_{j, k}^{(k-2)}\left(x_{j+k-1}\right) \Phi_{j+k-1, n}^{(n-k)}(x), \quad x \in\left[x_{j+k-1}, x_{j+k}\right] .
\end{aligned}
$$

Let now formula (12) be fulfilled in $\left[x_{j+l}, x_{j+l+1}\right]$ for some $l^{\prime}=l-1<k-2(l=1,2, \ldots, k-2)$. We must show its validity for $l \leqslant k-2$. According to $(10)$, and by the induction assumption for $x \in\left[x_{j+l}, x_{j+l+1}\right]$, we have

$$
\begin{aligned}
B_{j, k}(x)= & P_{j, l-1, k-2}\left(x_{j+l}\right) \\
& +B_{j, k}^{(k-2)}\left(x_{j+l-1}\right) \Phi_{j+l-1, n}^{(n-k)}\left(x_{j+l}\right)+B_{j, k}^{(k-2)}\left(x_{j+l}\right) \Psi_{j+l-1, n}^{(n-k)}\left(x_{j+l}\right) \\
& +\frac{1}{c_{j, k-1}} \int_{x_{j+l}}^{x} B_{j, k-1}(\tau) \mathrm{d} \tau-\frac{1}{c_{j+1, k-1}} \int_{x_{j+l}}^{x} B_{j+1, k-1}(\tau) \mathrm{d} \tau \\
= & P_{j, l-1, k-2}\left(x_{j+l}\right)+B_{j, k}^{(k-2)}\left(x_{j+l}\right) \Psi_{j+l-1, n}^{(n-k)}\left(x_{j+l}\right)+\frac{1}{c_{j, k-1}} \\
& \times\left\{\left.\sum_{l=1}^{l} B_{j, k^{\prime}}^{\left(k^{\prime}-2\right)}\left(x_{j+l^{\prime}}\right) \sum_{r=n-k^{\prime}}^{n-3} z_{j+l^{\prime}, n}^{(r)} \frac{\left(\tau-x_{j+l^{\prime}}\right)^{r-n+k^{\prime}+1}}{\left(r-n+k^{\prime}+1\right) !}\right|_{x_{j+l}} ^{x}\right. \\
& \left.+\left.B_{j, k^{\prime}}^{\left(k^{\prime}-2\right)}\left(x_{j+l}\right) \Phi_{j+l, n}^{\left(n-k^{\prime}-1\right)}(\tau)\right|_{x_{j+l}} ^{x}+\left.B_{j, k^{\prime}}^{\left(k^{\prime}-2\right)}\left(x_{j+l+1}\right) \Psi_{j+l, n}^{\left(n-k^{\prime}-1\right)}(\tau)\right|_{x_{j+l}} ^{x}\right\} \\
& -\frac{1}{c_{j+1, k-1}}\left\{I+\left.B_{j+1, k^{\prime}}^{\left(k^{\prime}-2\right)}\left(x_{j+l}\right) \Phi_{j+l, n}^{\left(n-k^{\prime}-1\right)}(\tau)\right|_{x_{j+l}} ^{x}+\left.B_{j+1, k^{\prime}}^{\left(k^{\prime}-2\right)}\left(x_{j+l+1}\right) \Psi_{j+l, n}^{\left(n-k^{\prime}-1\right)}(\tau)\right|_{x_{j+l}} ^{x}\right\},
\end{aligned}
$$

where

$$
\begin{aligned}
I & =\left.\sum_{l^{\prime}=1}^{l-1} B_{j+1, k^{\prime}}^{\left(k^{\prime}-2\right)}\left(x_{j+1+l^{\prime}}\right) \sum_{r=n-k^{\prime}}^{n-3} z_{j+1+l^{\prime}, n}^{(r)} \frac{\left(\tau-x_{j+1+l^{\prime}}\right)^{r-n+k^{\prime}+1}}{\left(r-n+k^{\prime}+1\right) !}\right|_{x_{j+l}} ^{x} \\
& =\left.\sum_{l^{\prime}=1}^{l} B_{j+1, k^{\prime}}^{\left(k^{\prime}-2\right)}\left(x_{j+l^{\prime}}\right) \sum_{r=n-k^{\prime}}^{n-3} z_{j+l^{\prime}, n}^{(r)} \frac{\left(\tau-x_{j+l^{\prime}}\right)^{r-n+k}}{(r-n+k) !}\right|_{x_{j+l}} ^{x} .
\end{aligned}
$$

Using the formula of differentiation (11) we obtain

$$
B_{j, k}^{(k-2)}\left(x_{j+l}\right)=B_{j, k^{\prime}}^{\left(k^{\prime}-2\right)}\left(x_{j+l}\right) / c_{j, k^{\prime}}-B_{j+1, k^{\prime}}^{\left(k^{\prime}-2\right)}\left(x_{j+l}\right) / c_{j+1, k^{\prime}} .
$$

This permits us to transform expression (16) into the form

$$
\begin{aligned}
B_{j, k}(x)= & \sum_{l^{\prime}=1}^{l-1} B_{j, k}^{(k-2)}\left(x_{j+l^{\prime}}\right) \sum_{r=n-k}^{n-3} z_{j+l^{\prime}, n}^{(r)} \frac{\left(x_{j+1}-x_{j+l^{\prime}}\right)^{r-n+k}}{(r-n+k) !} \\
& +B_{j, k}^{(k-2)}\left(x_{j+1}\right) z_{j+l, n}^{(n-k)}+B_{j, k}^{(k-2)}\left(x_{j+1}\right) \Phi_{j+l, n}^{(n-k)}(x)+B_{j, k}^{(k-2)}\left(x_{j+l+1}\right) \Psi_{j+l, n}^{(n-k)}(x)
\end{aligned}
$$




$$
\begin{aligned}
& +\sum_{l^{\prime}=1}^{l} B_{j, k}^{(k-2)}\left(x_{j+l^{\prime}}\right) \sum_{r=n-k^{\prime}}^{n-3} z_{j+l^{\prime}, n}^{(r)} \frac{\left(x-x_{j+l^{\prime}}\right)^{r-n+k}}{(r-n+k) !} \\
& -\sum_{l^{\prime}=1}^{l} B_{j, k}^{(k-2)}\left(x_{j+l^{\prime}}\right) \sum_{r=n-k^{\prime}}^{n-3} z_{j+l^{\prime}, n}^{(r)} \frac{\left(x_{j+1}-x_{j+l^{\prime}}\right)^{r-n+k}}{(r-n+k) !} \\
= & \sum_{l^{\prime}=1}^{l} B_{j, k}^{(k, 2)}\left(x_{j+l^{\prime}}\right) z_{j+l^{\prime}, n}^{(n)}+B_{j, k}^{(k)}{ }^{2)}\left(x_{j+1}\right) \Phi_{j+l, n}^{(n) k)}(x) \\
& +B_{j, k}^{(k-2)}\left(x_{j+l+1}\right) \Psi_{j+l, n}^{(n-k)}(x)+\sum_{l^{\prime}=1}^{l} B_{j, k}^{(k-2)}\left(x_{j+l^{\prime}}\right) \sum_{r-n-k^{\prime}}^{n-3} z_{j+l^{\prime}, n}^{(r)} \frac{\left(x-x_{j+l^{\prime}}\right)^{r-n+k}}{(r-n+k) !} \\
= & P_{j, l, k-2}(x)+B_{j, k}^{(k-2)}\left(x_{j+1}\right) \Phi_{j+l, n}^{(n-k)}(x)+B_{j, k}^{(k-2)}\left(x_{j+l+1}\right) \Psi_{j+l, n}^{(n-k)}(x) .
\end{aligned}
$$

We have now proved formula (12) with

$$
P_{j, l, k-2}(x)=\sum_{l^{\prime}=1}^{l} B_{j, k}^{(k-2)}\left(x_{j+l^{\prime}}\right) \sum_{r=n-k}^{n-3} z_{j+l^{\prime}, n}^{(r)} \frac{\left(x-x_{j+l^{\prime}}\right)^{r-n+k}}{(r-n+k) !}, \quad l=1, \ldots, k-2 .
$$

Taking into account the conditions for continuity we obtain the validity of the formula (17) for $l=k-1$. However, according to (15), $P_{j, k-1, k-2} \equiv 0$. So from (17) for $l=k-1$ we obtain the identity (14). By subtracting this identity from (17) we arrive at the second formula in (13). This proves the theorem.

To use formulae (12) and (13) for calculations we first need to find the quantities $B_{j, k}^{(k-2)}\left(x_{j+1}\right)$, $l=1, \ldots, k-1 ; k=3, \ldots, n$. According to $(11)$,

$$
B_{j, k}^{(k-2)}\left(x_{j+1}\right)=B_{j, k-1}^{(k-3)}\left(x_{j+1}\right) / c_{j, k-1}-B_{j+1, k-1}^{(k-3)}\left(x_{j+1}\right) / c_{j+1, k-1}, \quad l=1, \ldots, k-1, k=3, \ldots, n .
$$

In particular, it follows from here with $B_{j, 2}\left(x_{j+1}\right)=1$ that

$$
\begin{gathered}
B_{j, 3}^{\prime}\left(x_{j+1}\right)=\frac{1}{c_{j, 2}}, \quad B_{j, 4}^{\prime \prime}\left(x_{j+1}\right)=\frac{1}{c_{j, 2} c_{j, 3}}, \\
B_{j, 3}^{\prime}\left(x_{j+2}\right)=-\frac{1}{c_{j+1,2}}, \quad B_{j, 4}^{\prime \prime}\left(x_{j+2}\right)=-\frac{1}{c_{j+1,2}}\left(\frac{1}{c_{j, 3}}+\frac{1}{c_{j+1,3}}\right), \\
B_{j, 4}^{\prime \prime}\left(x_{j+3}\right)=\frac{1}{c_{j+2,2} c_{j+1,3}},
\end{gathered}
$$

etc. Therefore, to find the required values of the derivatives of the GB-splines in the interior nodes of their support intervals, it is necessary to know the quantities $c_{j, k}$, i.e., the integrals of the GB-splines $B_{j, k}, k=2, \ldots, n-1$.

Theorem 3.2. The integrals $c_{j, k}=\int_{x_{j}}^{x_{j+k}} B_{j, k}(\tau) \mathrm{d} \tau$ of $G B$-splines are given by the formula

$$
c_{j, k}=\sum_{l=1}^{k-1} B_{j, k}^{(k-2)}\left(x_{j+1}\right) \sum_{r=n-k-1}^{n-3} z_{j+l, n}^{(r)} \frac{\left(x_{j+\alpha}-x_{j+1}\right)^{r-n+k+1}}{(r-n+k+1) !}, \quad \alpha=1, \ldots, k-1, k=2, \ldots, n-1 .
$$


Proof. For $k=2$ according to (9) we obtain

$$
c_{j, 2}=\int_{x_{j}}^{x_{j+2}} B_{j, 2}(\tau) \mathrm{d} \tau=B_{j, 2}\left(x_{j+1}\right) z_{j+1, n}^{(n-3)}, \quad B_{j, 2}\left(x_{j+1}\right)=1,
$$

which corresponds to the formula (19). Let us suppose by induction that the formula (19) holds for all $k^{\prime}=k-1<n-1(k=3, \ldots, n-1)$. We must prove its validity for $k^{\prime}+1=k \leqslant n-1$. By formula (12),

$$
\begin{aligned}
c_{j, k}= & \int_{x_{j}}^{x_{j+k}} B_{j, k}(\tau) \mathrm{d} \tau=B_{j, k}^{(k-2)}\left(x_{j+1}\right) \Psi_{j, n}^{(n-k-1)}\left(x_{j+1}\right) \\
& +\sum_{l=1}^{k-2} \int_{x_{j+1}}^{x_{j+l+1}}\left[P_{j, l, k-2}(\tau)+B_{j, k}^{(k-2)}\left(x_{j+1}\right) \Phi_{j+l, n}^{(n-k)}(\tau)\right. \\
& \left.+B_{j, k}^{(k-2)}\left(x_{j+l+1}\right) \Psi_{j+l, n}^{(n-k)}(\tau)\right] d \tau-B_{j, k}^{(k-2)}\left(x_{j+k-1}\right) \Phi_{j+k-1, n}^{(n-k-1)}\left(x_{j+k-1}\right) .
\end{aligned}
$$

Choosing the $\operatorname{knot} x_{j+\alpha} \in \operatorname{supp} B_{j, k}, 1 \leqslant \alpha \leqslant k-1$ and using formulae (13) we can transform expression (20) into the form

$$
\begin{aligned}
c_{j, k}= & \sum_{l=1}^{k-1} B_{j, k}^{(k-2)}\left(x_{j+1}\right) z_{j+1, n}^{(n-k-1)}+\left.\sum_{l=1}^{\alpha-1}\left[\sum_{l^{\prime}=1}^{l} B_{j, k}^{(k-2)}\left(x_{j+l^{\prime}}\right) \sum_{r=n-k}^{n-3} z_{j+l^{\prime}, n}^{(r)} \frac{\left(\tau-x_{j+l^{\prime}}\right)^{r-n+k+1}}{(r-n+k+1) !}\right]\right|_{x_{j+1}} ^{x_{j+l+1}} \\
& +\left.\sum_{l=\alpha}^{k-2}\left[-\sum_{l^{\prime}=l+1}^{k-1} B_{j, k}^{(k-2)}\left(x_{j+l^{\prime}}\right) \sum_{r=n-k}^{n-3} z_{j+l^{\prime}, n}^{(r)} \frac{\left(\tau-x_{j+l^{\prime}}\right)^{r-n+k+1}}{(r-n+k+1) !}\right]\right|_{x_{j+1}} ^{x_{j+l+1}} .
\end{aligned}
$$

Collecting here the terms with $B_{j, k}^{(k-2)}\left(x_{j+1}\right), l=1, \ldots, k-1$, we have

$$
\begin{aligned}
c_{j, k}= & \sum_{l=1}^{k-1} B_{j, k}^{(k-2)}\left(x_{j+1}\right) z_{j+1, n}^{(n-k-1)}+\sum_{l=1}^{\alpha-1} B_{j, k}^{(k-2)}\left(x_{j+1}\right) \sum_{r=n-k}^{n-3} z_{j+l, n}^{(r)} \frac{\left(x_{j+\alpha}-x_{j+1}\right)^{r} n|k| 1}{(r-n+k+1) !} \\
& +\sum_{l=\alpha+1}^{k-1} B_{j, k}^{(k-2)}\left(x_{j+1}\right) \sum_{r=n-k}^{n-3} z_{j+l, n}^{(r)} \frac{\left(x_{j+\alpha}-x_{j+1}\right)^{r-n+k+1}}{(r-n+k+1) !} \\
= & \sum_{l=1}^{k-1} B_{j, k}^{(k-2)}\left(x_{j+1}\right) \sum_{r=n-k-1}^{n-3} z_{j+l, n}^{(r)} \frac{\left(x_{j+\alpha}-x_{j+1}\right)^{r-n+k+1}}{(r-n+k+1) !}
\end{aligned}
$$

This proves the theorem.

Theorem 3.3. If $c_{j, k}, k=2, \ldots, n-1$, are integrals of $G B$-splines $B_{j, k}$ then the following equalities are valid:

$$
\begin{aligned}
& \sum_{l=1}^{k-1} B_{j, k}^{(k-2)}\left(x_{j+1}\right) \sum_{r=n-k-1+\alpha}^{n-3} z_{j+l, n}^{(r)} \frac{\left(-x_{j+1}\right)^{r-n+k+1-\alpha}}{(r-n+k+1-\alpha) !}=c_{j, k} \cdot \delta_{0, \alpha} \\
& \quad \alpha=0, \ldots, k-2, k=2, \ldots, n-2,
\end{aligned}
$$

where $\delta_{0, \alpha}$ is the Kronecker symbol. 
Proof. We can write these identities

$$
c_{j, k} \equiv F_{j, k}(x)=\sum_{l=1}^{k-1} B_{j, k}^{(k-2)}\left(x_{j+1}\right) \sum_{r=n-k-1}^{n-3} z_{j+l, n}^{(r)} \frac{\left(x-x_{j+1}\right)^{r-n+k+1}}{(r-n+k+1) !}, \quad k=2, \ldots, n-1 .
$$

For $k=2$, formula (22) does not depend on $x$ and coincides with (19). According to Theorem 3.2, the polynomial $F_{j, k}-c_{j, k}, 2 \leqslant k \leqslant n-1$, of order $k-1$ takes zero values at the points $x_{j+\alpha}, \alpha=$ $1, \ldots, k-1$. Therefore, by the Fundamental Theorem of Algebra it must be identically equal to zero.

Using the expansion of polynomials in (22) by powers of $x$ we obtain

$$
c_{j, k}=\sum_{l=1}^{k-1} B_{j, k}^{(k-2)}\left(x_{j+1}\right) \sum_{\alpha=0}^{k-2} \frac{x^{\alpha}}{\alpha !} \sum_{r=n-k-1+\alpha}^{n-3} z_{j+l, n}^{(r)} \frac{\left(-x_{j+1}\right)^{r-n+k+1-\alpha}}{(r-n+k+1-\alpha) !}, \quad k=2, \ldots, n-1 .
$$

The right-hand side is a polynomial of order $k-1$ for fixed $j, k$ while the left-hand side is constant. It follows that the coefficient of $x^{\alpha}$ equals $c_{j, k}$ when $\alpha=0$ and equals zero otherwise. From this we obtain the equalities (21). This proves the theorem.

To construct the GB-spline $B_{j, k}, k=3, \ldots, n$, we can formulate an algorithm by applying formulae (18) and (19), and requiring the calculation of the following quantities for GB-splines:

$$
\begin{array}{lcccc}
B_{j, 2}\left(x_{j+1}\right) & \cdots & B_{j, k}^{(k-2)}\left(x_{j+\alpha}\right) & \cdots & B_{j, n}^{(n-2)}\left(x_{j+\alpha}\right) \\
\vdots & \vdots & \vdots & \ddots & (\alpha=1, \ldots, n-1) \\
B_{j+n-k, 2}\left(x_{j+n-k+1}\right) & \cdots & B_{j+n-k, k}^{(k-2)}\left(x_{j+n-k+\alpha}\right) & & \\
\vdots & \ddots & (\alpha=1, \ldots, k-1) & & \\
B_{j+n-2,2}\left(x_{j+n}\right) & & & &
\end{array}
$$

and the integrals of GB-splines

$$
\begin{array}{ccccc}
c_{j, 2} & \cdots & c_{j, k} & \cdots & c_{j, n-1} \\
c_{j+1,2} & \cdots & c_{j+1, k} & \cdots & c_{j+1, n-1} \\
\vdots & \vdots & \vdots & \ddots & \\
c_{j+n-k, 2} & \cdots & c_{j+n-k, k} & & \\
\vdots & \ddots & & & \\
c_{j+n-2,2} . & & & &
\end{array}
$$

Algorithm 1. (a) Form the diagonal matrix $A=\left\{a_{i j}\right\}, i, j=1, \ldots, n-1$, with diagonal elements $a_{l+1, l+1}=B_{j+l, 1}\left(x_{j+l+2}\right)=1, l=0, \ldots, n-2$. Attach to the matrix $A$ at the left an additional column with elements $a_{l+1,0}=c_{j+l, 2}, l=0, \ldots, n-2$ calculated by formula (19).

(b) For $k=3, \ldots, n$, using formula (18) we find the elements $a_{\alpha, l+1}=B_{j+l-(k-2), k}^{(k-2)}\left(x_{j+\alpha}\right), \alpha=l+$ $3-k, \ldots, l+1, l=n-2, \ldots, k-2$, and place them on the main diagonal and on the first $k-2$ upper off-diagonals of the matrix $A$. At every step $k$ (for $k<n-1$ ) we also calculate the elements $a_{l+1, k-2}=c_{j+l-(k-2), k}, l=n-2, \ldots, k-2$, using formula (19) and place them into the $(k-2)$ nd column of the lower triangular part of the matrix $A$. 
As a result the matrix $A$ is transformed to the form

$$
A=\left(\begin{array}{cccccc}
c_{j, 2} & B_{j, 2}\left(x_{j+1}\right) & \ldots & B_{j, k}^{(k-2)}\left(x_{j+1}\right) & \cdots & B_{j, n}^{(n-2)}\left(x_{j+1}\right) \\
\vdots & \vdots & & \vdots & & \vdots \\
c_{j+k-2,2} & c_{j+k-3,3} & \cdots & B_{j, k}^{(k-2)}\left(x_{j+k-1}\right) & \cdots & B_{j, n}^{(n-2)}\left(x_{j+k-1}\right) \\
\vdots & \vdots & & \vdots & & \vdots \\
c_{j+n-2,2} & c_{j+n-3,3} & \cdots & c_{j+n-1-k, k+1} & \cdots & B_{j, n}^{(n-2)}\left(x_{j+n-1}\right)
\end{array}\right)
$$

The $(k-1)$ st column of the upper triangular part of the matrix $A$ contains the quantities $B_{j, k}^{(k-2)}\left(x_{j+\alpha}\right)$, $\alpha=1, \ldots, k-1, k=2, \ldots, n$. This permits us to construct the GB-splines $B_{j, k}, k=2, \ldots, n$, using formulae (12) and (13). The integrals for these GB-splines are located along the main diagonal of the matrix $A$.

The supports of the GB-splines $B_{j, k}, k=2, \ldots, n$, begin at the point $x_{j}$. We can also consider an alternative version of the above algorithm in which the GB-splines $B_{j+k, n-k}, k=n-2, \ldots, 0$, whose supports end at the point $x_{j+n}$ are calculated.

Algorithm 2. (a) Form the diagonal matrix $A$ of dimension $(n-1) \times(n-1)$ with diagonal elements $a_{l+1, l+1}=B_{j+l, 2}\left(x_{j+l+1}\right)=1, l=0, \ldots, n-2$. Attach to $A$ the additional $(n)$ th row with elements $a_{n, l+1}=c_{j+l, 2}, l=0, \ldots, n-2$, calculated by formula (19).

(b) For $k=3, \ldots, n, l=0, \ldots, n-k$, we find $a_{l+1, l+\alpha}=B_{j+l, k}^{(k-2)}\left(x_{j+l+\alpha}\right), \alpha=k-1, \ldots, 1$ and (for $k<n-1) a_{n-k+2, l+1}=c_{j+l, k}$ by formulae (18) and (19).

As a result the matrix $A$ takes the form

$$
A=\left(\begin{array}{ccccc}
B_{j, n}^{(n-2)}\left(x_{j+1}\right) & \cdots & B_{j, n}^{(n-2)}\left(x_{j+1+n-k}\right) & \cdots & B_{j, n}^{(n-2)}\left(x_{j+n-1}\right) \\
\vdots & & \vdots & & \vdots \\
c_{j, k+1} & \cdots & B_{j+n-k, k}^{(k-2)}\left(x_{j+1+n-k}\right) & \cdots & B_{j+n-k, k}^{(k-2)}\left(x_{j+n-1}\right) \\
\vdots & & \vdots & & \vdots \\
c_{j, 3} & \cdots & c_{j+n-k, 3} & \cdots & B_{j+n-2,2}\left(x_{j+n-1}\right) \\
c_{j, 2} & \cdots & c_{j+n-k, 2} & \cdots & c_{j+n-2,2}
\end{array}\right) .
$$

The elements of the $(n-k+1)$ th row in the upper triangular part of the matrix $A$ permit us to construct the GB-splines $B_{j+n-k, k}, k=2, \ldots, n$, using formulae (12) and (13). The integrals of these GB-splines are located along the main diagonal of the matrix $A$.

\section{Another representation for GB-splines}

According to (12) and (13) the expressions for $B_{j, k}, k=3, \ldots, n$, in the subintervals $\left[x_{j+l-1}, x_{j+1}\right]$ and $\left[x_{j+1}, x_{j+l+1}\right]$ differ by the quantity

$$
\begin{aligned}
& -\Phi_{j+l-1, n}^{(n-k)}(x) B_{j, k}^{(k-2)}\left(x_{j+l-1}\right)+\left[\Phi_{j+l, n}^{(n-k)}(x)-\Psi_{j+l-l, n}^{(n-k)}(x)+\sum_{r=n-k}^{n-3} z_{j+l, n}^{(r)} \frac{\left(x-x_{j+1}\right)^{r-n-k}}{(r-n+k) !}\right] B_{j, k}^{(k-2)}\left(x_{j+1}\right) \\
& \quad+\Psi_{j+l, n}^{(n-k)}(x) B_{j, k}^{(k-2)}\left(x_{j+l+1}\right) .
\end{aligned}
$$


By summing over the jumps we arrive at the representation

$$
B_{j, k}(x)=\sum_{l=1}^{k-1} \Omega_{j+l, k}(x) B_{j, k}^{(k-2)}\left(x_{j+1}\right), \quad k=3, \ldots, n
$$

with

$$
\begin{aligned}
\Omega_{j+l, k}(x)= & \Psi_{j+l-1, n}^{(n-k)}(x) \theta\left(x-x_{j+l-1}\right)+\left[\Phi_{j+l, n}^{(n-k)}(x)-\Psi_{j+l-1, n}^{(n-k)}(x)+\sum_{r-n-k}^{n-3} z_{j+l, n}^{(r)} \frac{\left(x-x_{j+1}\right)^{r-n+k}}{(r-n+k) !}\right] \\
& \times \theta\left(x-x_{j+1}\right)-\Phi_{j+l, n}^{(n-k)}(x) \theta\left(x-x_{j+l+1}\right), \\
\theta(x-y)= & \begin{cases}1, & x \geqslant y, \\
0, & x<y .\end{cases}
\end{aligned}
$$

As $\theta(x-y)=1-\theta(y-x)$ we obtain

$$
B_{j, k}(x)=-\sum_{l=1}^{k-1} \hat{\Omega}_{j+l, k}(x) B_{j, k}^{(k-2)}\left(x_{j+1}\right)+R_{j, k}(x),
$$

where $\hat{\Omega}_{j+l, k}$ is derived from $\Omega_{j+l, k}$ by replacing $\theta\left(x-x_{j+m}\right)$ with $\theta\left(x_{j+m}-x\right), m=l-1, l, l+1$. Now according to (14)

$$
R_{j, k}(x)=\sum_{l=1}^{k-1} B_{j, k}^{(k-2)}\left(x_{j+1}\right) \sum_{r=n-k}^{n-3} z_{j+l, n}^{(r)} \frac{\left(x-x_{j+1}\right)^{r-n+k}}{(r-n+k) !} \equiv 0, \quad k=3, \ldots, n
$$

and it follows from formulae (23) and (25) that $B_{j, k}(x) \equiv 0$ if $x \notin\left(x_{j}, x_{j+k}\right)$. Any of these formulae can be used to define the GB-spline of order $k, 3 \leqslant k \leqslant n$.

We will transform the expression for the function $\Omega_{j+l, k}$ in (24). Using the Taylor expansion of the functions $\Phi_{j+l, n}^{(n-k)}, \Psi_{j+l, n}^{(n-k)}$ with the remainder in integral form and the properties (2) we have

$$
\begin{aligned}
\Omega_{j+l, k}(x)= & {\left[\int_{x_{j+l-1}}^{x} \frac{(x-\tau)^{k-2}}{(k-2) !} \Psi_{j+l-1, n}^{(n-1)}(\tau) d \tau\right] \theta\left(x-x_{j+l-1}\right) } \\
& +\left[\int_{x_{j+1}}^{x} \frac{(x-\tau)^{k-2}}{(k-2) !} \Phi_{j+l, n}^{(n-1)}(\tau) \mathrm{d} \tau \int_{x_{j+1}}^{x} \frac{(x-\tau)^{k-2}}{(k-2) !} \Psi_{j+l-1, n}^{(n-1)}(\tau) \mathrm{d} \tau\right] \\
& \times \theta\left(x-x_{j+1}\right)-\left[\int_{x_{j+l+1}}^{x} \frac{(x-\tau)^{k-2}}{(k-2) !} \Phi_{j+l, n}^{(n-1)}(\tau) \mathrm{d} \tau\right] \theta\left(x-x_{j+l+1}\right) .
\end{aligned}
$$

From here we obtain for polynomial splines with

$$
\Phi_{j, n}(x)=-\frac{\left(x-x_{j+1}\right)^{n-1}}{(n-1) ! h_{j}}, \quad \Psi_{j, n}(x)=\frac{\left(x-x_{j}\right)^{n-1}}{(n-1) ! h_{j}}
$$


that

$$
\begin{aligned}
& \Omega_{m, k}(x)=\left(x_{m+1}-x_{m-1}\right) g_{k}\left[x ; x_{m-1}, x_{m}, x_{m+1}\right], \quad m=j+l, \\
& g_{k}(x, y)=(-1)^{k}(x-y)_{+}^{k-1} /(k-1) !, \quad z_{+}=\max (0, z)
\end{aligned}
$$

and thus (23) is transformed to

$$
B_{j, k}(x)=\left(x_{j+k}-x_{j}\right) g_{k}\left[x ; x_{j}, \ldots, x_{j+k}\right], \quad k=3, \ldots, n .
$$

The recurrence formula (10) takes the form [14]

$$
B_{j, k}(x)=\frac{x-x_{j}}{x_{j+k-1}-x_{j}} B_{j, k-1}(x)+\frac{x_{j+k}-x}{x_{j+k}-x_{j+1}} B_{j+1, k-1}(x)
$$

with

$$
c_{j, k}=\int_{x_{j}}^{x_{j+k}} B_{j, k}(x) \mathrm{d} x=\frac{x_{j+k}-x_{j}}{k} .
$$

\section{Properties of GB-splines}

Let us formulate some properties of GB-splines which are similar to those of polynomial B-splines [14].

Theorem 5.1. The functions $B_{j, k}, k=2, \ldots, n$, have the following properties:

(i) $B_{j, k}(x)>0$ if $x \in\left(x_{j}, x_{j+k}\right)$ and $B_{j, k}(x) \equiv 0$ if $x \notin\left(x_{j}, x_{j+k}\right)$;

(ii) the splines $B_{j, k}$ have $k-2$ continuous derivatives;

(iii) for $k \geqslant 3$ and $x \in[a, b], \sum_{j=-k+1}^{N-1} B_{j, k}(x)=1$;

(iv) for $x \in\left[x_{j}, x_{j+1}\right]$,

$$
\begin{array}{r}
\Psi_{j, n}^{(r)}(x)=\left(\prod_{k=2}^{n-r-1} c_{j, k}\right) B_{j, n-r}(x), \quad \Phi_{j, n}^{(r)}(x)=\prod_{k=2}^{n-r-1}\left(-c_{j-k+1, k}\right) B_{j-n+1+r, n-r}(x) \\
j=0, \ldots, N-1, r=0, \ldots, n-2, \quad \text { where } c_{j, k}=\int_{x_{j}}^{x_{j+k}} B_{j, k}(\tau) \mathrm{d} \tau .
\end{array}
$$

Proof. The functions $B_{j+\alpha, 2}$ given by formula (9) are positive if $x \in\left(x_{j+\alpha}, x_{j+\alpha+2}\right)$, while $B_{j+\alpha, 2}(x)=0$ if $x \notin\left(x_{j+\alpha}, x_{j+\alpha+2}\right)$, and are monotone on the intervals $\left[x_{j+\alpha+1}, x_{j+\alpha+l+1}\right], l=0,1 ; \alpha=0, \ldots, k-2$.

Let us suppose by induction that $B_{j+\alpha, k-1}(x)>0$ if $x \in\left(x_{j+\alpha}, x_{j+\alpha+k-1}\right), B_{j+\alpha, k-1} \equiv 0$ if $x \notin\left(x_{j+\alpha}\right.$, $\left.x_{j+\alpha+k-1}\right)$ and $B_{j+\alpha, k-1}^{(k-3)}(x)$ is monotone on the intervals $\left[x_{j+\alpha+l}, x_{j+\alpha+l+1}\right], l=0, \ldots, k-2$ with $(-1)^{l+1} B_{j+\alpha, k-1}^{(k-3)}\left(x_{j+\alpha+l}\right)>0, l=1, \ldots, k-2, \alpha=0,1$. Then by formula (11) the function $B_{j, k}^{(k-2)}$ is monotone in $\left[x_{j+l}, x_{j+l+1}\right], l=0, \ldots, k-1$, and in addition $(-1)^{l+1} B_{j, k}^{(k-2)}\left(x_{j+l}\right)>0, l=1, \ldots, k-1$. Therefore, $B_{j, k}^{(k-2)}$ has exactly $k-2$ zeros in $\left(x_{j}, x_{j+k}\right)$ and by Rolle's theorem, $B_{j, k}$ does not vanish on $\left(x_{j}, x_{j+k}\right)$. Taking formula (10) into account, we have $B_{j, k}(x)>0$ if $x \in\left(x_{j}, x_{j+k}\right)$ and $B_{j, k}(x)=0$ if $x \notin\left(x_{j}, x_{j+k}\right)$. 
Property (ii) is obvious by virtue of the recurrence formula (10) and by continuity of the function $B_{j, 2}$.

According to (12) for $x \in\left[x_{j}, x_{j+1}\right]$,

$$
B_{j, n}(x)=B_{j, n}^{(n-2)}\left(x_{j+1}\right) \Psi_{j, n}(x), B_{j-n+1, n}(x)=B_{j-n+1, n}^{(n-2)}\left(x_{j}\right) \Phi_{j, n}(x) .
$$

Applying the differentiation formula (11) we obtain

$$
\begin{aligned}
& B_{j, n}^{(r)}(x)=\prod_{k=1}^{r} c_{j, n-k}^{-1} B_{j, n-r}(x), \\
& B_{j-n+1, n}^{(r)}(x)=\prod_{k=1}^{r}\left(-c_{j-n+1+k, n-k}^{-1}\right) B_{j-n+1+r, n-r}(x), \quad x \in\left[x_{j}, x_{j+1}\right], \quad r=1,2, \ldots, n-2
\end{aligned}
$$

and in particular,

$$
B_{j, n}^{(n-2)}\left(x_{j+1}\right)=\prod_{k=1}^{n-2} c_{j, n-k}^{-1}, \quad B_{j-n+1, n}^{(n-2)}\left(x_{j}\right)=\prod_{k=1}^{n-2}\left(-c_{j-k, k+1}^{-1}\right) .
$$

Therefore, if $x \in\left[x_{j}, x_{j+1}\right]$ then according to (26)-(28) we have

$$
\Psi_{j, n}^{(r)}(x)=\prod_{k=2}^{n-r-1} c_{j, k} B_{j, n-r}(x), \quad \Phi_{j, n}^{(r)}(x)=\prod_{k=2}^{n-r-1}\left(-c_{j-k+1, k}\right) B_{j-n+1+r, n-r}(x), \quad r=0, \ldots, n-2 .
$$

Applying the recurrence formula (10) for $k>2$, we have for $x \in[a, b]$ :

$$
\begin{aligned}
\sum_{j--k+1}^{N-1} B_{j, k}(x) & =\sum_{j=-k+1}^{N-1}\left[\int_{x_{j}}^{x} \frac{B_{j, k-1}(\tau)}{c_{j, k-1}} \mathrm{~d} \tau-\int_{x_{j+1}}^{x} \frac{B_{j+1, k-1}(\tau)}{c_{j+1, k-1}} \mathrm{~d} \tau\right] \\
& =\int_{x_{-k+1}}^{x} \frac{B_{-k+1, k-1}(\tau)}{c_{-k+1, k-1}} \mathrm{~d} \tau-\int_{x_{N}}^{x} \frac{B_{N, k-1}(\tau)}{c_{N, k-1}} \mathrm{~d} \tau=\int_{x_{-k+1}}^{x_{0}} \frac{B_{-k+1, k-1}(\tau)}{c_{-k+1, k-1}} \mathrm{~d} \tau=1,
\end{aligned}
$$

i.e.,

$$
\sum_{j=-k+1}^{N-1} B_{j, k+1}(x) \equiv 1, \quad k=3, \ldots, n \text { if } x \in[a, b] .
$$

This proves the theorem.

Corollary 5.2. The following identities are valid:

$$
\sum_{j=i-k+2}^{i-1} \sum_{l=1}^{i-j} B_{j, k}^{(k-2)}\left(x_{j+l}\right) \sum_{r=n-k}^{n-3} z_{j+l, n}^{(r)} \frac{\left(x-x_{j+l}\right)^{r-n+k}}{(r-n+k) !} \equiv 1, \quad k=3, \ldots, n .
$$


Proof. Substituting formula (12) into the identity (29) written for $x \in\left[x_{i}, x_{i+1}\right]$, we obtain

$$
\begin{aligned}
\sum_{j=i-k+1}^{i} B_{j, k}(x)= & \Phi_{i, n}^{(n-k)}(x) \sum_{j=i-k+1}^{i-1} B_{j, k}^{(k-2)}\left(x_{i}\right)+\Psi_{i, n}^{(n-k)}(x) \sum_{j=i-k+2}^{i} B_{j, k}^{(k-2)}\left(x_{i+1}\right) \\
& +\sum_{j=i-k+2}^{i-1} P_{j, i-j, k-2}(x) \equiv 1 .
\end{aligned}
$$

According to (29)

$$
\sum_{j=i-k+1}^{i-1} B_{j, k}^{(k-2)}\left(x_{i}\right)=\sum_{j=i-k+2}^{i} B_{j, k}^{(k-2)}\left(x_{i+1}\right)=0 .
$$

Then using (13) one obtains

$$
\sum_{j=i-k+2}^{i-1} P_{j, i-j, k-2}(x)=\sum_{j=i-k+2}^{i-1} \sum_{l=1}^{i-j} B_{j, k}^{(k-2)}\left(x_{j+l}\right) \sum_{r=n-k}^{n-3} z_{j+l, n}^{(r)} \frac{\left(x-x_{j+l}\right)^{r-n+k}}{(r-n+k) !} \equiv 1 .
$$

This proves the corollary.

Corollary 5.3. Let $F_{j, k}, k=2, \ldots, n-1$, be polynomials as defined in (22). Then the following equalities are valid:

$$
\sum_{j=-k+1}^{N-1} \int_{u}^{b} B_{j, k}(x) \mathrm{d} x=\sum_{j=-k+1}^{N-1} F_{j, k}(x)=b-a, \quad k=2, \ldots, n-1 .
$$

Proof. Integrating the identity (29) on the interval $[a, b]$ and using (22) we obtain

$$
\begin{aligned}
\sum_{j=-k+1}^{N-1} \int_{a}^{b} B_{j, k}(x) \mathrm{d} x & =\sum_{j=-k+1}^{N-1} \int_{x_{j}}^{x_{j+k+1}} B_{j, k}(x) \mathrm{d} x \\
& =\sum_{j=-k+1}^{N-1} c_{j, k}=\sum_{j=-k+1}^{N-1} F_{j, k}(x)=b-a .
\end{aligned}
$$

This proves the corollary.

Theorem 5.4. The $G B$-splines $B_{j, k}, k=2, \ldots, n$, have supports of minimum length.

Proof. It follows from the explicit formula (9) that the support of the GB-spline $B_{j, 2}$ cannot be reduced. Let us suppose that the assertion of the theorem is fulfilled for some $k^{\prime}=k-1<n$ ( $k=$ $3, \ldots, n)$. Using mathematical induction we will prove its validity for $k^{\prime}+1=k \leqslant n$.

By the properties of the functions $\Phi_{j \mid l, n}, \Psi_{j \mid l, n}, 0 \leqslant l \leqslant k-1$, and by formula (12), a GB-spline $B_{j, k}$ cannot be different from zero on only a part of the subinterval $\left[x_{j+l}, x_{j+1+l}\right], 0 \leqslant l \leqslant k-1$. If we suppose that $B_{j, k}$ is zero on interval $\left[x_{j+l}, x_{j+1+l}\right], 0 \leqslant l \leqslant k-1$, then due to the continuity of $B_{j, k}^{(k-2)}$, we have $B_{j, k}^{(k-2)}\left(x_{j+l}\right)=B_{j, k}^{(k-2)}\left(x_{j+l+1}\right)=0$. 
Using formula (11) one can show, however, that $(-1)^{l+1} B_{j, k}^{(k-2)}\left(x_{j+l}\right)>0, l=1, \ldots, k-1$. For $k=2$ we have $B_{j, 2}\left(x_{j+1}\right)=1$. Suppose by induction that $(-1)^{l+1} B_{j, k^{\prime}}^{\left(k^{\prime}-2\right)}\left(x_{j+l}\right)>0, l=1, \ldots, k^{\prime}-1$, for $1 \leqslant k^{\prime} \leqslant k-1$. By formula (11) we get

$$
\begin{aligned}
(-1)^{l+1} B_{j, k}^{(k-2)}\left(x_{j+l}\right) & =(-1)^{l+1}\left[\frac{B_{j, k^{\prime}}^{\left(k^{\prime}-2\right)}\left(x_{j+l}\right)}{c_{j, k^{\prime}}}-\frac{B_{j+1, k^{\prime}}^{\left(k^{\prime}-2\right)}\left(x_{j+l}\right)}{c_{j+1, k^{\prime}}}\right] \\
& =(-1)^{l+1} \frac{B_{j, k^{\prime}}^{\left(k^{\prime}-2\right)}\left(x_{j+l}\right)}{c_{j, k^{\prime}}}+(-1)^{l} \frac{B_{j+1, k^{\prime}}^{\left(k^{\prime}-2\right)}\left(x_{j+1+(l-1)}\right)}{c_{j+1, k^{\prime}}}>0, \quad l=1, \ldots, k-1 .
\end{aligned}
$$

We have obtained a contradiction. This proves the theorem.

Theorem 5.5. The GB-splines $B_{j, k}, j=-k+1, \ldots, N-1, k=2, \ldots, n$, are linearly independent and form a basis for the space $S_{k}^{\mathrm{G}}$ of generalized splines.

Proof. Let us assume to the contrary that there exist constants $b_{j, k}, j=-k+1, \ldots, N-1, k=2, \ldots, n$, which are not all equal to zero and such that

$$
b_{-k+1, k} B_{-k+1, k}(x)+\cdots+b_{N-1, k} B_{N-1, k+1}(x)=0, \quad x \in[a, b] .
$$

According to formula (9), and taking into account the properties of the functions $\Phi_{i, n}$ and $\Psi_{i, n}$ in (2), we obtain from (30) for $k=2$

$$
\sum_{j=-1}^{N-1} b_{j, 2} B_{j, 2}\left(x_{i}\right)=b_{i-1,2}=0, \quad i=0, \ldots, N .
$$

Thus, $b_{j, 2}=0, j=-1, \ldots, N-1$, and the functions $B_{j, 2}, j=-1, \ldots, N-1$, are linearly independent.

Suppose by induction that the functions $B_{j, k^{\prime}}, j=-k^{\prime}, \ldots, N-1$ are linearly independent for some $k^{\prime}=k-1<n(k=3, \ldots, n)$. We will prove the assertion of theorem for $k^{\prime}+1=k \leqslant n$. Differentiating the equality (30) and using the recurrence formula (10) we have

$$
\begin{aligned}
\sum_{j=-k+1}^{N-1} b_{j, k} B_{j, k}^{\prime}(x) & =\sum_{j=-k+1}^{N-1} b_{j, k}\left[\frac{B_{j, k-1}(x)}{c_{j, k-1}}-\frac{B_{j+1, k}(x)}{c_{j+1, k-1}}\right] \\
& =b_{-k+1, k} \frac{B_{-k+1, k-1}(x)}{c_{-k+1, k-1}}+\sum_{j=-k+2}^{N-1}\left(b_{j, k}-b_{j-1, k}\right) \frac{B_{j, k-1}(x)}{c_{j, k-1}}-b_{N-1, k} \frac{B_{N, k-1}(x)}{c_{N, k-1}}=0 .
\end{aligned}
$$

The supports of the GB-splines $B_{j, k-1}, j=-k+1, N$, however, are outside the interval $[a, b]$. By the induction assumption, the GB-splines $B_{j, k-1}, j=-k+2, \ldots, N-1$ are linearly independent and thus from (31) we get $b_{j, k}-b_{j-1, k}=0, j=-k+2, \ldots, N-1$ or $b_{j, k}=c=$ const, $j=-k+1, \ldots, N-1$. By Theorem 5.1, the GB-splines $B_{j, k}, j=-k+1, \ldots, N-1$ give a partition of unity on the interval $[a, b]$. Using this property, and Eq. (29), we arrive at the equality

$$
\sum_{j=-k+1}^{N-1} b_{j, k} B_{j, k}(x)=c \sum_{j=-k+1}^{N-1} B_{j, k}(x)=c \cdot 1=0 .
$$


Therefore, $b_{j, k}=0, j=-k+1, \ldots, N-1$ and the GB-splines $B_{j, k}, j=-k+1, \ldots, N-1, k=2, \ldots, n$, are linearly independent.

Since by Definition 2.1, $\operatorname{dim}\left(S_{k}^{\mathrm{G}}\right)=(k) N-(k-1)(N-1)=N+k-1$, we see that the GB-splines $B_{j, k} \in S_{k}^{\mathrm{G}}, j=-k+1, \ldots, N-1$ form a basis of this space. This proves the theorem.

By virtue of this theorem, any spline $S \in S_{k}^{\mathrm{G}}, k=2, \ldots, n$, can be uniquely written in the form

$$
S(x)=\sum_{j=-k+1}^{N-1} b_{j, k} B_{j, k}(x) \quad \text { for } x \in[a, b]
$$

for some constant coefficients $b_{j, k}$.

Corollary 5.6. Any spline $S \not \equiv 0$ in $S_{k}^{\mathrm{G}}, k=2, \ldots, n$, with finite support of minimal length coincides with a GB-spline up to a constant multiplier.

Proof. By Theorem 5.4 the minimal support of a spline $S \in S_{k}^{\mathrm{G}}, k=2, \ldots, n$ different from identical zero, is an interval $\left(x_{i}, x_{i+k}\right), i=0, \ldots, N-k$. Using representation (32) we get

$$
S(x)=b_{i-k+1, k} B_{i-k+1, k}+\cdots+b_{i+k-1, k} B_{i+k-1, k}(x) .
$$

As $S \equiv 0$ for $x \notin\left(x_{i}, x_{i+k}\right)$, when choosing sequentially $x \in\left(x_{p}, x_{p+1}\right), p=i-k+1, \ldots, i-1$, we obtain $b_{p, k}=0$. In the same manner, $b_{p, k}=0$ for $p=i+k-1, \ldots, i+1$. Therefore, $S(x)=b_{i, k} B_{i, k}(x)$. This proves the corollary.

\section{Series of GB-splines}

In practical applications such as approximation of functions, discrete data etc., one considers linear combinations of GB-splines. According to Theorem 5.5, any generalized spline $S \in S_{k}^{\mathrm{G}}, k=2, \ldots, n$, can be uniquely represented in the form

$$
S(x)=\sum_{j=-k+1}^{N-1} b_{j, k} B_{j, k}(x) \text { for } x \in[a, b]
$$

with some constant coefficients $b_{j, k}$.

Let us study how the behaviour of a spline $S$ depends on the coefficients $b_{j, k}$. Since GB-splines are local, from (33) we obtain the inequalities

$$
\min _{i-k+1 \leqslant j \leqslant i} b_{j, k} \leqslant S(x)=\sum_{j=i-k+1}^{i} b_{j, k} B_{j, k}(x) \leqslant \max _{i-k+1 \leqslant j \leqslant i} b_{j, k}, \quad x_{i} \leqslant x \leqslant x_{i+1}, \quad k=2, \ldots, n .
$$

Hence it follows that the behaviour of the spline $S$ on the interval $\left[x_{i}, x_{i+1}\right]$ is determined by the coefficients $b_{i-k+1, k}, \ldots, b_{i, k}$. In particular, in order for a spline $S$ to be zero at a point of the interval $\left[x_{i}, x_{i+1}\right]$, it is necessary that $b_{j, k} b_{j+1, k}<0$ for some $i-k+1 \leqslant j \leqslant i$. 
The estimate (34) can be substantially improved on. Applying the differentiation formula (11), we obtain for $r \leqslant k-2$

$$
S^{(r)}(x)=\sum_{j=-k+r+1}^{N-1} b_{j, k}^{(r)} B_{j, k-r}(x),
$$

where

$$
b_{j, k}^{(l)}= \begin{cases}b_{j, k}, & l=0, \\ \frac{b_{j, k}^{(l-1)}-b_{j-1, k}^{(l-1)}}{c_{j, k-l}}, & l=1,2, \ldots, r .\end{cases}
$$

Lemma 6.1. If $b_{j, k} \geqslant 0(\leqslant 0), j=-k+1, \ldots, N-1 ; k=2, \ldots, n$, then $S(x) \geqslant 0(\leqslant 0)$ for all $x$.

The conclusion is obvious, because the GB-splines $B_{j, k}$ are nonnegative.

Lemma 6.2. If $b_{j, k}>b_{j-1, k}\left(b_{j, k}<b_{j-1, k}\right), j=-k+2, \ldots, N-1, k=3, \ldots, n$, then the function $S$ is monotonically increasing (decreasing).

Proof. According to formulae (35) and (36), we have

$$
S^{\prime}(x)=\sum_{j=-k+2}^{N-1} b_{j, k}^{(1)} B_{j, k-1}(x), \quad b_{j, k}^{(1)}=\frac{b_{j, k}-b_{j-1, k}}{c_{j, k-1}} .
$$

Because the GB-splines $B_{j, k-1}, k=3, \ldots, n$, are nonnegative, the formula above and Lemma 1 imply that $S$ is monotonic. This proves the lemma.

Lemma 6.3. If $b_{j, k}^{(1)}>b_{j-1, k}^{(1)}\left(b_{j, k}^{(1)}<b_{j-1, k}^{(1)}\right), j=-k+3, \ldots, N-1 ; k=4, \ldots, n$, then the function $S$ is convex downwards (upwards).

Proof. By virtue of (35) and (36), we have

$$
S^{\prime \prime}(x)=\sum_{j=-k+3}^{N-1} b_{j, k}^{(2)} B_{j, k-2}(x), \quad b_{j, k}^{(2)}=\frac{b_{j, k}^{(1)}-b_{j-1, k}^{(1)}}{c_{j, k-2}} .
$$

Because the GB-splines $B_{j, k-2}, k=4, \ldots, n$, are nonnegative, taking into account Lemma 1 we obtain that $S$ is convex. This proves the lemma.

Let $Z_{[a, b]}(f)$ denote the number of isolated zeros of a function $f$ on the interval $[a, b]$.

Lemma 6.4. If the spline $S(x)=\sum_{j=-k}^{N-1} b_{j, k} B_{j, k}(x), k=2, \ldots, n$, is not identically zero on any subinterval of $[a, b]$, then

$$
Z_{[a, b]}(S) \leqslant N+k-2 \text {. }
$$


Proof. According to (35) and (9), for $x \in\left[x_{i}, x_{i+1}\right]$, we have

$$
S^{(k-2)}(x)=\sum_{j=-1}^{N-1} b_{j, k}^{(k-2)} B_{j, 2}(x)=b_{i-1, k}^{(k-2)} \Phi_{i, n}^{(n-2)}(x)+b_{i, k}^{(k-2)} \Psi_{i, n}^{(n-2)}(x) .
$$

This function has at most one zero on $\left[x_{i}, x_{i+1}\right]$, because the functions $\Phi_{i, n}^{(n-2)}$ and $\Psi_{i, n}^{(n-2)}$ are monotonous and nonnegative on this subinterval. Hence $Z_{[a, b]}\left(S^{(k-2)}\right) \leqslant N$. Then, according to Rolle's theorem [14], we find $Z_{[a, b]}(S) \leqslant N+k-2$. This proves the lemma.

Denote by $\operatorname{supp} B_{j, k}=\left\{x \mid B_{j, k}(x) \neq 0\right\}, k=2, \ldots, n$, the support of GB-spline $B_{j, k}$, i.e. the interval $\left(x_{j}, x_{j+k}\right)$.

Theorem 6.5. Assume that $\tau_{-k+1}<\tau_{-k+2}<\cdots<\tau_{N-1}, k=2, \ldots, n$. Then

$$
D=\operatorname{det}\left(B_{j, k}\left(\tau_{i}\right)\right) \neq 0, \quad i, j=-k+1, \ldots, N-1
$$

if and only if

$$
\tau_{j} \in \operatorname{supp} B_{j, k}, \quad j=-k+1, \ldots, N+1 .
$$

If condition (38) is satisfied, then $D>0$.

Proof. Let us prove the theorem by induction. It is clear that the theorem holds for a single basis function. Assume that it also holds for $l-1$ basis functions. Let us show that if (38) is satisfied, then $D \neq 0$ for $l$ basis functions.

Let $\tau_{l} \notin \operatorname{supp} B_{l, k}$. If $\tau_{l}$ lies to the left (right) with respect to the support of $B_{l, k}$ then the last column (line) of the determinant $D$ consists of zeros, i.e., $D=0$. If $\tau_{l} \in \operatorname{supp} B_{l, k}$ and $D=0$, then there exists a nonzero vector $c=\left(c_{-k+1, k}, \ldots, c_{l-k, k}\right)$ such that

$$
S\left(\tau_{p}\right)=\sum_{j=-k+1}^{l-k} c_{j, k} B_{j, k}\left(\tau_{p}\right)=0, \quad p=-k+1, \ldots, l-k,
$$

i.e., the spline $S$ has $l$ isolated zeros. But this contradicts Lemma 6.4, which states that $S$ can have no more than $l-1$ isolated zeros. Hence $c=0$ and $D \neq 0$.

Now it only remains to prove that $D>0$ if (38) is satisfied. Let us choose $x_{p}<\tau_{p}<x_{p+1}$ for all $p$. Then the diagonal elements of $D$ are positive and all the elements above the main diagonal are zero, i.e., $D>0$. It is clear that $D$ depends continuously on $\tau_{p}, p=-k+1, \ldots, l-k$, and $D \neq 0$ for $\tau_{p} \in \operatorname{supp} B_{p, k}$. Hence the determinant $D$ is positive if condition (38) is satisfied. This proves the theorem.

The following three statements follow immediately from Theorem 6.5.

Corollary 6.6. The system of $G B$-splines $\left\{B_{j, k}\right\}, j=-k+1, \ldots, N-1, k-2, \ldots, n$, is a weak Chebyshevian system in the sense of [6], i.e., for any $\tau_{-k+1}<\tau_{-k+2}<\cdots<\tau_{N-1}$ we have $D \geqslant 0$, and $D>0$ if and only if condition (38) is satisfied. If the latter is satisfied, then the generalized spline $S(x)=\sum_{j=-k+1}^{N-1} b_{j, k} B_{j, k}(x), k=2, \ldots, n$, has no more than $N+k-2$ isolated zeros. 
Corollary 6.7. If the conditions of Theorem 6.5 are satisfied, the solution of the interpolation problem

$$
S\left(\tau_{i}\right)=f_{i}, \quad i=-k+1, \ldots, N-1, \quad f_{i} \in \mathbb{R}
$$

exists and is unique.

Let $A=\left\{a_{i j}\right\}, i=1, \ldots, m, j=1, \ldots, n$, be a rectangular $(m \times n)$ matrix with $m \leqslant n$. The matrix $A$ is said to be totally nonnegative (totally positive) [4] if the minors of all orders of the matrix are nonnegative (positive), i.e., for all $1 \leqslant l \leqslant m$ we have

$$
\operatorname{det}\left(a_{i_{p} j_{q}}\right) \geqslant 0(>0) \text { for all } 1 \leqslant i_{1}<\cdots<i_{l} \leqslant m, 1 \leqslant j_{1}<\cdots<j_{l} \leqslant n .
$$

Corollary 6.8. For arbitrary integers $-k+1 \leqslant v_{-k+1}<\cdots<v_{l-k} \leqslant N-1$ and $\tau_{-k+1}<\tau_{-k+2}<\cdots$ $<\tau_{l-k}, k=2, \ldots, n$, we have

$$
D_{l}=\operatorname{det}\left\{B_{v_{j}, k}\left(\tau_{i}\right)\right\} \geqslant 0, \quad i, j=-k+1, \ldots, l-k,
$$

and $D_{l}>0$ if and only if

$$
\tau_{j} \in \operatorname{supp} B_{v_{j}, k}, \quad j=-k+1, \ldots, l-k
$$

i.e., the matrix $\left\{B_{j, k}\left(\tau_{i}\right)\right\}, i, j=-k+1, \ldots, N-1$ is totally nonnegative.

The last statement is proven by induction on the basis of Theorem 6.5 and the recurrence relations for the minors of the matrices $\left\{B_{j, k}\left(\tau_{i}\right)\right\}, k=2, \ldots, n$. The proof does not differ from that described by Schumaker [14].

Since the supports of GB-splines are compact, the matrix of the system (39) is a banded matrix and has $2 k-1$ nonzero diagonals in general. If the knots of the spline $x_{i}, i=-k+1, \ldots, N-1$, are placed in a suitable manner, then the number of nonzero diagonals of this matrix can be reduced to $k-1$.

De Boor and Pinkus [2] proved that linear systems with totally nonnegative matrices can be solved by Gaussian elimination without choosing a pivot element. Thus, the system (39) can be solved efficiently by the conventional Gauss method.

Denote by $S^{-}(\boldsymbol{v})$ the number of sign changes (variations) in the sequence of components of the vector $v=\left(v_{1}, \ldots, v_{n}\right)$, with zero being neglected. Karlin [6] showed that if a matrix $A$ is totally nonnegative, then it decreases the variation, i.e.

$$
S^{-}(A \boldsymbol{v}) \leqslant S(\boldsymbol{v}) \text {. }
$$

By virtue of Corollary 6.8 , the totally nonnegative matrix $\left\{B_{j, k}\left(\tau_{i}\right)\right\}, i, j=-k+1, \ldots, N-1, k=$ $2, \ldots, n$, formed by the GB-splines decreases the variation.

For a bounded real function $f$, let $S^{-}(f)$ be the number of sign changes of the function $f$ on the real axis $\mathbb{R}$ without taking into account the zeros

$$
S^{-}(f)=\sup _{p} S^{-}\left[f\left(\tau_{1}\right), \ldots, f\left(\tau_{p}\right)\right], \quad \tau_{1}<\tau_{2}<\cdots<\tau_{p}
$$


Theorem 6.9. The generalized spline $S(x)=\sum_{j=-k+1}^{N-1} b_{j, k} B_{j, k}(x), k=2, \ldots, n$, is a variation diminishing function, i.e., the number of sign changes of $S$ does not exceed the one in the sequence of its coefficients

$$
S_{\mathbb{R}}^{-}\left(\sum_{j=-k+1}^{N-1} b_{j, k} B_{j, k}\right) \leqslant S^{-}(\boldsymbol{b}), \quad \boldsymbol{b}=\left(b_{-k+1, k}, \ldots, b_{N-1, k}\right) .
$$

Proof. We use the approach proposed by Schumaker [14]. Let $S^{-}(\boldsymbol{b})=d-1$. Let us divide the coefficients $b_{j, k}$ into $d$ groups:

$$
b_{-k+1, k}, \ldots b_{k_{2}, k}, b_{k_{2}+1, k}, \ldots, b_{k_{3}, k}, \ldots, b_{k_{d}+1, k}, \ldots, b_{N-1, k} .
$$

In each group at least one coefficient is not zero, and all the nonzero coefficients have the same sign.

Putting $k_{1}=-k$ and $k_{d+1}=N-1$, we define the function

$$
\tilde{B}_{j, k}(x)=\sum_{i=k_{j}+1}^{k_{j+1}}\left|b_{i, k}\right| B_{i, k}(x), \quad j=1, \ldots, d .
$$

Then for arbitrary $\tau_{1}<\tau_{2}<\cdots<\tau_{d}$ we have

$$
\begin{gathered}
\operatorname{det}\left(\tilde{B}_{j, k}\left(\tau_{i}\right)\right)_{i, j=1}^{d}=\sum_{v_{1}=k_{1}+1}^{k_{2}} \cdots \sum_{v_{d}=k_{d}+1}^{k_{d+1}}\left|b_{v_{1}, k}\right| \cdots\left|b_{v_{d}, k}\right| \operatorname{det}\left(B_{j, k}\left(\tau_{i}\right)\right) \geqslant 0, \\
i=1, \ldots, d, j=v_{1}, \ldots, v_{d}, k=2, \ldots, n
\end{gathered}
$$

by virtue of Corollary 6.8 and because at least one coefficient $b_{i, k}$ is not zero in each group. It is clear that we can choose $\tau_{1}<\tau_{2}<\cdots<\tau_{d}$ such that $\operatorname{det}\left(\tilde{B}_{j, k}\left(\tau_{i}\right)\right)>0$. Hence the functions $\tilde{B}_{j, k}$ are linearly independent.

Assume that $\delta= \pm 1$ is the sign of the first group of the coefficients $b_{i, k}$. Let us take $\tilde{b}_{i, k}=(-1)^{i-1} \delta$, $i=1,2, \ldots, d$. Then

$$
\tilde{S}(x)=\sum_{i=1}^{d} \tilde{b}_{i, k} \tilde{B}_{i, k}(x) \equiv S(x)=\sum_{j=-k+1}^{N-1} b_{j, k} B_{j, k}(x) .
$$

Applying Lemma 6.4, we obtain

$$
Z\left(\sum_{j=-k+1}^{N-1} b_{j, k} B_{j, k}\right)=Z\left(\sum_{i=1}^{d} \tilde{b}_{i, k} B_{i, k}\right) \leqslant d-1=S^{-}\left(b_{-k+1, k}, \ldots, b_{N-1, k}\right), \quad k=2, \ldots, n .
$$

This proves the theorem.

The statement of Theorem 6.9 can be refined, namely we can point out a relation between the point at which the spline changes its sign and the corresponding spline coefficient. The coefficient corresponds to the GB-spline whose support includes the point of the sign change [see (34)]. 
Theorem 6.10. Assume that the inequalities $(-1)^{i} S\left(\tau_{i}\right)>0, i=1,2, \ldots, d$, are valid for the generalized spline $S(x)=\sum_{j=-k+1}^{N-1} b_{j, k} B_{j, k}(x)$ at some $\tau_{1}<\tau_{2}<\cdots<\tau_{d}$. Then there exist $-k+1 \leqslant j_{1}<j_{2}$ $<\cdots<j_{d} \leqslant N-1$ such that

$$
(-1)^{i} b_{j_{i}, k} B_{j_{i}, k}\left(\tau_{i}\right)>0, \quad i=1, \ldots, d \text {. }
$$

The proof of this statement does not differ from the proof of the corresponding theorem for polynomial B-splines [14].

\section{Invariance of generalized splines with respect to affine transformations}

In some applications of spline approximation we encounter affine transformations of the independent variable: $\hat{x}=p x+q$, where $p \neq 0$ and $q$ are constant. It is well-known that the usual Lagrange-Newton, Chebyshev, etc., polynomials are invariant with respect to such transformations. Let us show that generalized splines also have this property.

Let $\hat{S}_{n}^{\mathrm{G}}$ be a set of generalized splines on the mesh $\hat{\Delta}=\left\{\hat{x}_{i} \mid \hat{x}_{i}=p x_{i}+q, i=0, \ldots, N\right\}$ which is obtained from the linear space $S_{n}^{\mathrm{G}}$ by affine transformation of the variable $x$.

Theorem 7.1. An approximating generalized spline $S \in S_{n}^{\mathrm{G}}$ is invariant with respect to affine transformations of the real axis $\mathbb{R}=(-\infty, \infty)$.

Proof. The function $B_{j, 2}$ in (9) can be written in the form

$$
B_{j, 2}(x)= \begin{cases}\psi_{j, n}^{(n-2)}\left(\frac{x-x_{j}}{h_{j}}\right), & x_{j} \leqslant x \leqslant x_{j+1} \\ \varphi_{j+1, n}^{(n-2)}\left(\frac{x-x_{j+1}}{h_{j+1}}\right), & x_{j+1} \leqslant x \leqslant x_{j+2} \\ 0 & \text { otherwise }\end{cases}
$$

where

$$
\psi_{j, n}\left(\frac{x-x_{j}}{h_{j}}\right) h_{j}^{n-2}=\Psi_{j, n}(x), \quad \varphi_{j+1, n}\left(\frac{x-x_{j+1}}{h_{j+1}}\right) h_{j+1}^{n-2}=\Phi_{j+1, n}(x) .
$$

Using the change of the variable $\hat{x}=p x+q$ we get

$$
\begin{aligned}
& \psi_{j, n}^{(n-2)}\left(\frac{x-x_{j}}{h_{j}}\right)=\psi_{j, n}^{(n-2)}\left(\frac{\hat{x}-\hat{x}_{j}}{\hat{x}_{j+1}-\hat{x}_{j}}\right), \\
& \varphi_{j+1, n}^{(n-2)}\left(\frac{x-x_{j+1}}{h_{j+1}}\right)=\varphi_{j+1, n}^{(n-2)}\left(\frac{\hat{x}-\hat{x}_{j+1}}{\hat{x}_{j+2}-\hat{x}_{j+1}}\right) .
\end{aligned}
$$


Therefore, $B_{j, 2}(x)=\hat{B}_{j, 2}(\hat{x})$. Let the equality $B_{j, l}(x)=\hat{B}_{j, l}(\hat{x})$ be fulfilled for $l=k-1<n(k=3, \ldots, n)$. By virtue of the recurrence relation (10) we have

$$
B_{j, k}(x)=\int_{x_{j}}^{x} \frac{B_{j, k-1}(\tau)}{c_{j, k-1}} \mathrm{~d} \tau-\int_{x_{j+1}}^{x} \frac{B_{j+1, k-1}(\tau)}{c_{j+1, k-1}} \mathrm{~d} \tau, \quad k=3, \ldots, n,
$$

with

$$
c_{j, k-1}=\int_{x_{j}}^{x_{j+k-1}} B_{j, k-1}(\tau) \mathrm{d} \tau .
$$

Using the substitution $\hat{\tau}=p \tau+q$ we obtain by induction

$$
\begin{aligned}
& c_{j, k-1}=\int_{\hat{x}_{j}}^{\hat{x}_{i+k-1}} \hat{B}_{j, k-1}(\hat{\tau}) \frac{1}{p} \mathrm{~d} \hat{\tau}=\frac{1}{p} \hat{c}_{j, k-1}, \\
& B_{j, k}(x)=\int_{\hat{x}_{j}}^{\hat{x}} \frac{\hat{B}_{j, k-1}(\hat{\tau})}{\hat{c}_{j, k-1}} \mathrm{~d} \hat{\tau}-\int_{\hat{x}_{j+1}}^{\hat{x}} \frac{\hat{B}_{j+1, k-1}(\hat{\tau})}{\hat{c}_{j+1, k-1}} \mathrm{~d} \hat{\tau}=\hat{B}_{j, k}(\hat{x}), \quad k=3, \ldots, n .
\end{aligned}
$$

If now $S \in S_{n}^{\mathrm{G}}$ and $\hat{S} \in \hat{S}_{n}^{\mathrm{G}}$ are approximating splines on the meshes $\Delta$ and $\hat{\Delta}$, respectively, connected by an affine transformation $\hat{x}=p x+q$, then by the uniqueness of the spline representation as a linear combination of GB-splines we obtain

$$
S(x)=\sum_{j} b_{j} B_{j, n}(x)=\sum_{j} b_{j} \hat{B}_{j, n}(\hat{x})=\hat{S}(\hat{x}) .
$$

Therefore, the approximating generalized spline $S$ is invariant with respect to affine transformations of its variable. This proves the theorem.

It was shown above that $B_{j, k}(x)=\hat{B}_{j, k}(\hat{x}), k=2, \ldots, n$. By differentiation of this equality we obtain

$$
\left.\frac{\mathrm{d}}{\mathrm{d} x}\left[B_{j, k}(x)\right]=\frac{\mathrm{d}}{\mathrm{d} x}\left[\hat{B}_{j, k}(\hat{x})\right]=\frac{\mathrm{d}}{\mathrm{d} \hat{x}}\left[\hat{B}_{j, k}(\hat{x})\right] \frac{\mathrm{d} \hat{x}}{\mathrm{~d} x}=p \hat{B}_{j, k}^{\prime}(\hat{x})\right], \quad k=3, \ldots, n .
$$

Differentiating now the equality (40), we can write down

$$
S^{\prime}(x)=\sum_{j} b_{j} B_{j, n}^{\prime}(x)=\sum_{j} b_{j} p \hat{B}_{j, n}^{\prime}(\hat{x})=p \hat{S}^{\prime}(\hat{x}) .
$$

By repeated differentiation of the last and next to last equalities we arrive at

$$
S^{(r)}(x)=\sum_{j} b_{j} B_{j, n}^{(r)}(x)=\sum_{j} b_{j} p^{r} \hat{B}_{j, n}^{(r)}(\hat{x})=p^{r} \hat{S}^{(r)}(\hat{x}), \quad r=1, \ldots, n-2 .
$$

\section{Local approximation by GB-splines}

Using the locality of GB-splines one can reduce the representation of a spline $S$ as a linear combination of GB-splines (33) for $k=n$ to the form

$$
S(x)=\sum_{j=i=n+1}^{i} b_{j, n} B_{j, n}(x), \quad x \in\left[x_{i}, x_{i+1}\right], \quad i=0,1, \ldots, N-1 .
$$


Theorem 8.1. The restriction (41) of the spline $S$ to the interval $\left[x_{i}, x_{i+1}\right]$ can be written in the form

$$
S(x)=P_{i, n-2}(x)+b_{i-1, n}^{(n-2)} \Phi_{i, n}(x)+b_{i, n}^{(n-2)} \Psi_{i, n}(x),
$$

where

$$
\begin{aligned}
& P_{i, n-2}(x)=\sum_{j=i-n+2}^{i-1} b_{j, n} \sum_{l^{\prime}=1}^{i-j} B_{j, n}^{(n-2)}\left(x_{j+l^{\prime}}\right) \sum_{r=0}^{n-3} z_{j+l^{\prime}, n}^{(r)} \frac{\left(x-x_{j+l^{\prime}}\right)^{r}}{r !} \\
& b_{j, n}^{(k)}=\frac{b_{j, n}^{(k-2)}-b_{j-1, n}^{(k-2)}}{c_{j, n-k}}, \quad k=1, \ldots, n-2, b_{j, n}^{(0)}=b_{j, n}, j=i-n+1, \ldots, i .
\end{aligned}
$$

Proof. We use induction on $n$. According to formula (9), the representation (42) holds for $n=2$ :

$$
S(x)=b_{i-1,2} B_{i-1,2}(x)+b_{i, 2} B_{i, 2}(x)=b_{i-1,2} \Phi_{i, 2}(x)+b_{i, 2} \Psi_{i, 2}(x), \quad x \in\left[x_{i}, x_{i+1}\right] .
$$

Suppose that (42) holds for $n=l-1$. Then for $n=l$ one has from (41)

$$
S(x)=\sum_{j=i-l+1}^{i} b_{j, l} B_{j, l}(x), \quad x \in\left[x_{i}, x_{i+1}\right] .
$$

Using (4) for $n=l$, one obtains

$$
\begin{aligned}
S(x) & =\sum_{j=i-l+1}^{i} b_{j, l}\left[P_{j, i-j, l-2}(x)+B_{j, l}^{(l-2)}\left(x_{i}\right) \Phi_{i, l}(x)+B_{j, l}^{(l-2)}\left(x_{i+1}\right) \Psi_{i, l}(x)\right] \\
& =P_{i, l-2}(x)+I,
\end{aligned}
$$

where according to (6)

$$
P_{i, l-2}(x)=\sum_{j=i-l+2}^{i-1} b_{j, l} \sum_{l^{\prime}=1}^{i-j} B_{j, l}^{(l-2)}\left(x_{j+l^{\prime}}\right) \sum_{r=0}^{l-3} z_{j+l^{\prime}, l}^{(r)} \frac{\left(x-x_{j+l^{\prime}}\right)^{r}}{r !}
$$

and

$$
I=\Phi_{i, l}(x) \sum_{j=i-l+1}^{i-1} b_{j, l} B_{j, l}^{(l-2)}\left(x_{i}\right)+\Psi_{i, l}(x) \sum_{j=i-l+2}^{i} b_{j, l} B_{j, l}^{(l-2)}\left(x_{i+1}\right) .
$$

By applying the formula of differentiation (11) one gets

$$
\begin{aligned}
I= & \Phi_{i, l}(x) \sum_{j=i-l+1}^{i-1} b_{j, l}\left[\frac{B_{j, l-2}^{(l-3)}\left(x_{i}\right)}{c_{j, l-1}}-\frac{B_{j+l, l-1}^{(l-3)}\left(x_{i}\right)}{c_{j+1, l-1}}\right] \\
& +\Psi_{i, l}(x) \sum_{j=i-l+2}^{i} b_{j, l}\left[\frac{B_{j, l-1}^{(l-3)}\left(x_{i+1}\right)}{c_{j, l-1}}-\frac{B_{j+1, l-1}^{(l-3)}\left(x_{i+1}\right)}{c_{j+1, l-1}}\right] \\
= & \Phi_{i, l}(x)\left[b_{i-l+1, l} \frac{B_{i-l, l-1}^{(l-3)}\left(x_{i}\right)}{c_{i-l+1, l-1}}+\sum_{j=i-l+2}^{i-1} \frac{b_{j, l}-b_{j-1, l}}{c_{j, l-1}} B_{j, l-1}^{(l-3)}\left(x_{i}\right)-b_{i-1, l} \frac{B_{i, l-1}^{(l-3)}\left(x_{i}\right)}{c_{i, l-1}}\right]
\end{aligned}
$$




$$
+\Psi_{i, l}(x)\left[b_{i-l+2, l} \frac{B_{i-l+2, l-1}^{(l-3)}\left(x_{i+1}\right)}{c_{i-l+2, l-1}}+\sum_{j=i-l+3}^{i} \frac{b_{j, l}-b_{j-1, l}}{c_{j, l-1}} B_{j, l-1}^{(l-3)}\left(x_{i+1}\right)-b_{i, l} \frac{B_{i+1, l-1}^{(l-3)}\left(x_{i+1}\right)}{c_{i+1, l-1}}\right] .
$$

Because $B_{i-l+l^{\prime}+1, l-1}^{(l-3)}\left(x_{i+l^{\prime}}\right)=B_{i+l^{\prime}, l-1}^{(l-3)}\left(x_{i+l^{\prime}}\right)=0, l^{\prime}=0,1$, expression (45) can be rewritten in the form

$$
I=\Phi_{i, l}(x) \sum_{j=i-l+2}^{i-1} b_{j, l}^{(1)} B_{j, l-1}^{(l-3)}\left(x_{i}\right)+\Psi_{i, l}(x) \sum_{j=i-l+3}^{i} b_{j, l}^{(1)} B_{j, l-1}^{(l-3)}\left(x_{i+1}\right)
$$

with

$$
b_{j, l}^{(1)}=\frac{b_{j, l}-b_{j-1, l}}{c_{j, l-1}}, \quad j=i-l+2, \ldots, i .
$$

By the assumption of induction however,

$$
\sum_{j=i-(l-2)+l^{\prime}}^{i-1+l^{\prime}} b_{j, l-1} B_{j, l-1}^{(l-3)}\left(x_{i+l^{\prime}}\right)=b_{i-1+l^{\prime}, l-1}^{(l-3)}, \quad l^{\prime}=0,1 .
$$

Therefore one gets

$$
I=b_{i-1, l}^{(l-2)} \Phi_{i, l}(x)+b_{i, l}^{(l-2)} \Psi_{i, l}(x)
$$

and using (44), we can write down (43) in the form

$$
S(x)=P_{i, l-2}(x)+b_{i-1, l}^{(l-2)} \Phi_{i, l}(x)+b_{i, l}^{(l-2)} \Psi_{i, l}(x) .
$$

This is formula (42) in the assertion of the theorem for $n=l$. This proves the theorem.

Theorem 8.2. In formula (42) the polynomial $P_{i, n-2}$ can be written in the form

$$
P_{i, n-2}(x)=\sum_{k=0}^{n-3} b_{i-n+2+k, n}^{(k)} Q_{i, n-2}^{(n-3-k)}(x)
$$

where

$$
\begin{aligned}
& Q_{i, n-2}^{(k)}(x)= \begin{cases}Q_{i, n-2}(x) / c_{i-2,1}, & k=0, \\
\frac{Q_{i-1, n-2}^{(k-1)}(x)-Q_{i, n-2}^{(k-1)}(x)}{c_{i-k-1, k+2}}, & k=1,2, \ldots, n-3,\end{cases} \\
& Q_{j, n-2}(x)=\sum_{l=0}^{n-3} z_{j, n}^{(r)} \frac{\left(x-x_{j}\right)^{r}}{r !}, \quad j=i-n+3, \ldots, i, \quad Q_{i, n-2}^{(n-3)}(x) \equiv 1 .
\end{aligned}
$$

This assertion is new even for polynomial splines, and can be proven by induction. 


\section{Examples}

Let us give examples of the defining functions $\Phi_{i, n}(x)$ and $\Psi_{i, n}(x)$ in (1) which are most commonly used. In the examples given below they depend on the parameters

$$
\begin{aligned}
& \Phi_{i, n}(x)=\varphi_{i, n}(t) h_{i}^{n-2}=\psi_{n}\left(p_{i}, 1-t\right)\left(-h_{i}\right)^{n-2}, \\
& \Psi_{i, n}(x)=\psi_{i, n}(t) h_{i}^{n-2}=\psi_{n}\left(q_{i}, t\right) h_{i}^{n-2},
\end{aligned}
$$

where $t=\left(x-x_{i}\right) / h_{i}$ and $0 \leqslant p_{i}, q_{i}<\infty$.

By Definition 2.1 the function $\psi_{i, n}(t)$ satisfies the conditions

$$
\psi_{i, n}^{(r)}(0)=0, \quad r=0, \ldots, n-2, \quad \psi_{i, n}^{(n-2)}(1)=1 .
$$

(1) Rational splines:

$$
\psi_{i, n}(t)=\frac{t^{n-1}}{(n-1) !} \frac{C_{1, n}\left(q_{i}\right)}{1+q_{t}(1-t)}, \quad C_{1, n}^{-1}=\sum_{j=0}^{n-2} C_{n-2}^{j} \frac{q_{i}^{j}}{j+1},
$$

where

$$
C_{n-2}^{j}=\left(\begin{array}{c}
n-2 \\
j
\end{array}\right)
$$

is the usual binomial coefficient.

(2) Exponential splines:

$$
\psi_{i, n}(t)=\frac{t^{n-1}}{(n-1) !} e^{-q_{i}(1-t)} C_{2, n}\left(q_{i}\right), \quad C_{2, n}^{-1}\left(q_{i}\right)=\sum_{j=0}^{n-2} C_{n-2}^{j} \frac{q_{i}^{j}}{(j+1) !} .
$$

(3) Hyperbolic splines:

$$
\psi_{i, n}(t)=\frac{\Theta_{i, n}(t)}{q_{i}^{n-2} \sinh \left(q_{i}\right)},
$$

where

$$
\Theta_{i, n}(t)= \begin{cases}\sinh \left(q_{i} t\right)-\sum_{j=0}^{m-2} \frac{\left(t q_{i}\right)^{2 j+1}}{(2 j+1) !} & \text { if } n=2 m \\ \cosh \left(q_{i} t\right)-\sum_{j=0}^{m-2} \frac{\left(t q_{i}\right)^{2 j}}{(2 j) !} & \text { if } n=2 m-1 .\end{cases}
$$

(4) Splines with additional nodes:

$$
\psi_{i, n}(t)=\frac{1+q_{i}}{(n-1) !}\left(t-\frac{q_{i}}{1+q_{i}}\right)_{i}^{n-1} \text {. }
$$

If we take $\alpha_{i}=\left(1+p_{i}\right)^{-1}$ and $\beta_{i}=1-\left(1+q_{i}\right)^{-1}$, then the points $x_{i 1}=x_{i}+\alpha_{i} h_{i}$ and $x_{i 2}=x_{i}+\beta_{i} h_{i}$ fix the positions of two additional nodes of the spline on the interval $\left[x_{i}, x_{i+1}\right]$. By moving them, we can go from a spline of the order $n$ to a spline of the order $n-2$.

The constants $C_{k, n}\left(q_{i}\right), k=1,2$, in the expressions for the function $\psi_{i, n}(t)$ above are calculated from the condition $\psi_{i, n}^{(n-2)}(1)=1$ in (46). 


\section{Acknowledgements}

Appreciation is rendered to the Thailand Research Fund for the financial support which has made this research possible.

\section{References}

[1] C. de Boor, A Practical Guide to Splines, Springer, New York, 1978.

[2] C. de Boor, A. Pinkus, Backward error analysis for totally positive linear systems, Numer. Math. 27 (1977) 485-490.

[3] N. Dyn, A. Ron, Recurrence relations for Tchebicheffian B-splines, J. Anal. Math. 51 (1988) 118-138.

[4] F.R. Gantmacher, The Theory of Matrices, vol. 1, Chelsea Publishing Company, New York, 1960.

[5] A. Gresbrand, A recurrence relation for rational B-splines with prescribed poles, preprint.

[6] S. Karlin, Total Positivity, vol. 1, Stanford University Press, Stanford, CA, 1968.

[7] P.E. Koch, T. Lyche, Exponential B-splines in tension, in: C.K. Chui, L.L. Schumaker, J.D. Ward (Eds.), Approximation Theory VI, vol. II, Academic Press, Boston, 1989, pp. 361-364.

[8] P.E. Koch, T. Lyche, Construction of exponential tension B-splines of arbitrary order, in: P.J. Laurent, A. Le Méhaute, L.L. Schumaker (Eds.), Curves and Surfaces, Academic Press, New York, 1991, pp. 255-258.

[9] P.E. Koch, T. Lyche, Interpolation with exponential B-splines in tension, in: G. Farin et al. (Eds.), Geometric Modelling, Computing/Supplementum, vol. 8, Springer, Wien, 1993 pp. 173-190.

[10] B.I. Ksasov, Local bases for generalized cubic splines, Russian J. Numer. Anal. Math. Modelling 10 (1995) 49-80.

[11] B.I. Ksasov, Shape preserving spline approximation via local algorithms, in: F. Fontanella, K. Jetter, P.J. Laurent (Eds.), Advanced Topics in Multivariate Approximation, World Scientific, Singapore, 1996, pp. 181-196,

[12] B.I. Kvasov, GB-splines and their properties, Ann. Numer. Math. 3 (1996) 139-149.

[13] T. Lyche, A recurrence relation for Chebyshevian B-splines, Constr. Approx. 1 (1985) 155-173.

[14] L.L. Schumaker, Spline Functions: Basic Theory, Wiley, New York, 1981.

[15] L.L. Schumaker, On recurrences for generalized B-splines, J. Approx. Theory 36 (1982) 16-31. 